\title{
BIOMASS DELIGNIFICATION WITH GREEN SOLVENTS TOWARDS LIGNIN VALORISATION: IONIC LIQUIDS VS DEEP EUTECTIC SOLVENTS
}

\author{
André M. da Costa Lopes \\ CECOLAB - Collaborative Laboratory Towards Circular Economy \\ R. Nossa Senhora da Conceição, 3405-155 Oliveira do Hospital, Portugal. \\ CICECO - Aveiro Institute of Materials, Department of Chemistry, University of Aveiro, \\ 3810-193 Aveiro, Portugal, andre.lopes@cecolab.pt / andremcl@ua.pt \\ https://orcid.org/0000-0001-8855-6406
}

Article history: Received 12 August 2021, Accepted 13 August 2021, Available online 13 August 2021

\begin{abstract}
The use of renewable resources as feedstocks to ensure the production of goods and commodities for society has been explored in the last decades to switch off the overexploited and pollutant fossil-based economy. Today there is a strong movement to set bioeconomy as priority, but there are still challenges and technical limitations that must be overcome in the first place, particularly on biomass fractionation. For biomass to be an appellative raw material, an efficient and sustainable separation of its major components must be achieved. On the other hand, the technology development for biomass valorisation must follow green chemistry practices towards ecofriendly processes, otherwise no environmental leverage over traditional petrochemical technologies will be acquired. In this context, the application of green solvents, such as ionic liquids (ILs) and deep eutectic solvents (DES), in biomass fractionation is envisaged as promising technology that encompasses not only efficiency and environmental benefits, but also selectivity, which is a crucial demand to undertake cascade processes at biorefinery level. In particular, this article briefly discusses the disruptive achievements upon the application of ILs and DES in biomass delignification step towards an effective and selective separation of lignin from polysaccharides. The different physicochemical properties of these solvents, their interactions with lignin and their delignification capacity will be scrutinized, while some highlights will be given to the important characteristics of isolated lignin fractions for further valorisation. The advantages and disadvantages between ILs and DES in biomass delignification will be contrasted as well along the article.
\end{abstract}

\section{Keywords}

lignin, lignocellulosic biomass, green solvents, biomass delignification, valorisation

\section{Introduction}

The growth and technological evolution of humanity have brought economic and environmental dilemmas with massive challenges to health and welfare of society. The deficient resource management is one of the major bottlenecks to be solved and has a colossal handicap on our biosphere. The intensive exploration of nonrenewable fossil resources in the last decades has increased $\mathrm{CO}_{2}$ emissions, destruction of ecosystems, pollution of rivers and oceans, deforestation and biodiversity threats to global levels that undermines sustainable development of future generations. The scientific evidence has shown that the point of no return can be nearby and drastic moves are urgent to decline this fate to our planet. In this sense, the use of renewable resources to replace fossil-based feedstocks is seen as priority to undercut a pollutant and non-sustainable growth and to move forward with bioeconomy, a more sustainable model of development. Bioeconomy emerges as an alternative approach that deals with the maximal exploitation of biomass and other bio-based resources as starting materials to produce energy, fuels, chemicals and materials fulfilling society needs. The idea is to cover efficient processes for biomass fractionation and conversion into these commodities by the so-called biorefineries, analogous to current petroleum refineries [1]. The industrial activities that convert every single fraction of crude oil into all the commodities we know today have been intensively developed and explored since the industrial revolution at $18^{\text {th }}$ century. On the contrary, the technological development and readiness upon biomass processing and valorisation is quite lower and to push it to similar levels is a major challenge expected for the next few years. Therefore, high investment and straightforward efforts are needed to leverage sustainable processes and innovative technologies for the conversion and valorisation of biological and renewable resources, including biomass [2]. 
As one of the most abundant renewable resources in the world, lignocellulosic biomass is found mainly as agriculture residues (e.g. wheat straw, corn stover, sugarcane bagasse, etc.), energy crops (e.g. switchgrass, elephant grass, miscanthus, etc.) and forestry materials (e.g. wood, tree branches, tree bark, etc.). In Europe, their availability exceeds 1 billion dry tonnes per year [3], thus their interest as primary resource and raw material in industrial processes is obviously high. The composition of lignocellulosic biomass is mostly devoted to three main macromolecular components, namely cellulose (40-50 wt\%), hemicelluloses (10-30wt\%) and lignin (15-35 wt\%)[4]. Cellulose is a semicrystalline homopolysaccharide constituted by cellobiose as repeating unit, while hemicelluloses represent amorphous heteropolysaccharides constituted by different hexoses, pentoses and uronic acids. In contrast to cellulose, the structure and chemical composition of hemicelluloses are variable and are dependent on their biomass source. Both cellulose and hemicelluloses compose the polysaccharide fraction of lignocellulosic biomass and play essentially structural roles. In contrast, lignin is a random aromatic polymer constituted by phenylpropanoid units and the most conventional ones are $p$-coumaryl, coniferyl and sinapyl alcohols [4]. Recently, other aromatic units, like flavonoids, hydroxystilbenes, among others, have been also reported to make part of lignin structure [5]. These units are randomly linked by several types of C-O (e.g. $\beta-0-4$ and $\alpha-0-4)$ and $C-C$ (e.g. $\beta-\beta, \beta-5$ and $\beta-1$ ) chemical bonds. Lignin not only gives structural rigidity to plant biomass, but also exhibits protective role against any external biota. Other fractions, such as proteins, phenolic, lipophilic and inorganic compounds also exist in lignocellulosic biomass, although at much lower content $(<5$ wt\%). The way biomass polysaccharides and lignin are linked (intermolecular and covalent bonds) leads to an intricate and complex three-dimensional matrix difficult to disrupt by means of any physical, chemical and/or biological treatment. This recalcitrance is one of the key aspects that hinders the profit in generating bioproducts from these raw materials and must be overcome with efficient fractionation processes. Indeed, there are still technological hurdles to achieve maximal exploitation of lignocellulosic biomass, while today the concerns related to the environmental impact of developed technologies must be taken into account as well.

The fractionation and isolation of biomass polysaccharides have been tackled for a long time. Pulp and paper manufacturing was the first industry to valorise lignocellulosic biomass since $19^{\text {th }}$ century [6]. The isolation of cellulose pulp by removing lignin from wood is the main focus of these industries to produce paper at different grades. Nowadays, there are several well-known pulp and paper processes at industrial scale, including Kraft, Soda and Sulfite pulping, that uses water and inorganic chemicals, like $\mathrm{NaOH}, \mathrm{Na}_{2} \mathrm{~S}, \mathrm{SO}_{2}$ and alkali metals, to enable wood delignification efficiently and to maintain cellulose fibres intact and at desired strength. Cellulose pulp has been used mostly in the manufacture of paper and carboards, but an increasing interest to produce novel cellulose-based materials to substitute petrochemical ones has been observed lately as a clear movement to increase sustainability [7]. This kind of strategy is expected to place pulp and paper industries in the path of the biorefinery concept and could be a model to follow in the bioeconomy activity. On the other hand, the interest in converting polysaccharides from agriculture residues and energy crops towards $2 \mathrm{G}$ bioethanol at industrial scale has been approached by few companies in the current century. In this case, hydrothermal (steam), acid (sulfuric), alkaline (ammonia) and organosolv (acetic acid, ethanol) treatments have been applied to lignocellulosic biomass to open up the biomass matrix, allowing for better accessibility of cellulolytic enzymes (celluloses and xylanases) towards polysaccharide hydrolysis into C5 and C6 sugars. The monosaccharide enriched hydrolysate is then used as feed substrate to microorganisms capable of converting those carbohydrates into ethanol. Other biotechnological approaches aiming at the conversion of C5 and C6 sugars into value added chemicals, including butanol, organic acids, among others has been approached, yet at lab and/or pilot scales.

Bearing this in mind, pulp and paper companies and 2G bioethanol industries have shared a similar principle: to raise the value of biomass carbohydrates. In their framework of activities, lignin is considered as by-product and today no real value has been extracted from it. In case of pulp and paper companies, the black liquor resulting from wood delignification is burnt in boilers to recover the initial inorganic chemicals. This operation enables the combustion of lignin and other organic fractions present in the black liquor to generate energy, which is directly supplied to the factory and/or sold to the grid [8]. Similar approach has been delivered by $2 \mathrm{G}$ ethanol industries, in which lignin can be incinerated in combined heat and power units [9]. Although such bio-based companies refer to those integrated processes as self-sufficient in terms of energy requirements, the long-term sustainability of this practice might be questioned. Lignin is an aromatic polymer and may represent between 20-35 wt\% of initial lignocellulosic feedstocks (wood or others). Therefore, as an isolated material, lignin has the potential to replace the aromatic fraction of fossil sources in the formulation of chemicals and materials. However, one of the drawbacks that has led to decline a higher valorisation of lignin rather than its combustion 
is the quality of isolated samples. For instance, sulfur-based reagents $\left(\mathrm{Na}_{2} \mathrm{~S}\right.$ and $\left.\mathrm{SO}_{2}\right)$ along with high temperatures (150-170 으) are often applied in pulp and paper making to achieve wood delignification. This energy intensive process enables the isolation of a low-value sulfur-based lignin with extensive chemical modification characterised by condensed macromolecules, high molecular dispersion and low number of functional groups (loss of functionality). These are clearly undesired characteristics for its further application hindering an appropriate valorisation.

The strategy for polysaccharide processing has been designed and refined over the years, but a lack of comprehensive valorisation of lignin has been noted. Biomass delignification processes should expose cellulose fibres intact (pulp and paper activity) or accessible to enzymes (biotechnological industry), but simultaneously produce high-quality lignin with chemical and structural features as close as possible to native. Low molecular dispersion and high degree of functional groups, including hydroxyl and carboxylic groups, are desired for the fractionated lignin. Functional and less condensed lignin will be more prone for chemical reaction either in its cleavage to value added monomeric aromatic compounds [10] or in the synthesis of new bio-based materials [11]. Therefore, it is reasonable that efforts to develop new biomass fractionation processes are crucial in this research. The main strategy may stand on the priority to isolate polysaccharides with simultaneous separation of high-quality lignin fraction. However, there is also the "lignin-first" approach, where processes aim directly at the isolation/conversion of lignin [12]. A major example of the lignin-first approach is the reductive cleavage fractionation (RCF) technology that comprises solvent-based lignin extraction with integrated conversion in the presence of a transition metal under hydrogen atmosphere or assisted with a hydrogen-donor solvent or another reducing agent [12].

Independently of the main target, polysaccharides or lignin, a sustainable use of lignocellulosic biomass still relies on the adequate valorisation of each macromolecular component. In this regard, efficiency and selectivity on the separation/fractionation of cellulose, hemicellulose and lignin are important criteria to evaluate the sustainability of current and new biomass valorisation processes. Furthermore, the environmental impact of developed technologies must be assessed, where the application of environmentally friendly tools (solvents, catalysts, reagents, etc.) must be encouraged and coupled with low-energy requirements. In this sense, green solvents have rose as key tools to enable biomass fractionation at mild conditions. Ionic liquids (ILs) and deep eutectic solvents (DES) have been elected as major players, especially on the biomass delignification step. The isolated lignin has exhibited interesting physicochemical properties for further application, being one of the advantages of these new technologies against conventional processes mentioned above.

This article briefly reveals some of the ground-breaking scientific achievements in biomass delignification with ILs and DES. The definition and physicochemical properties of these solvents are quite important to be described and distinguished beforehand to understand the mechanistic insights upon their biomass delignification performance. Afterwards, their delignification ability and why isolated lignin discloses favourable properties for further valorisation will be discussed as well.

Ionic liquids vs Deep Eutectic Solvents: definition and properties

ILs and DES have been claimed as green solvents and interesting alternatives to traditional solvents. Due to their wide range of physicochemical properties, ILs and DES have been applied in many fields of research, such as chemical catalysis and separation, pharmaceutical and cosmetic formulations, biological applications, $\mathrm{CO}_{2}$ sequestration, fuel desulfurization, biomass fractionation and conversion, among others. Although ILs and DES possess similar applications and share some characteristics, their differences must be stressed out and clarified, including definition, chemical composition and physicochemical properties.

ILs have been discovered more than 100 years ago, when Paul Walden mixed nitric acid with ethanolamine to obtain ethylammonium nitrate $\left(\left[\mathrm{EtNH}_{3}\right]\left[\mathrm{NO}_{3}\right]\right)$ with the melting point of $12{ }^{\circ} \mathrm{C}$ [13]. Since then, the research on ILs and their applications have been expanded exponentially. By definition, ILs are salts composed of an asymmetric and large organic cation coupled with an inorganic or organic anion, exhibiting a melting point $<100$ ${ }^{\circ} \mathrm{C}$ [13]. The low melting point is a consequence of weaker interactions established between ILs' cation and anion when compared to strong ionic bonds existing in ordinary salts, like sodium chloride, which melts at $801{ }^{\circ} \mathrm{C}$. Furthermore, categorization between protic ionic liquids (PILs) and aprotic ionic liquids (AILs) has been adopted. PILs disclose the advantage of being easier to prepare as their synthesis rely on a simple proton transference from a Brønsted acid to a Brønsted base. In this case, chemical equilibrium between ions and molecular 
precursors are established. The higher the difference between acid and base pka $(\Delta \mathrm{pKa})$, the higher is the ionization of PIL. On the other hand, the synthesis of AILs includes different synthetic strategies, as for instance, the Menshutkin reaction that enables the formation of a quaternary positive charged ammonium from neutral amine species. Moreover, AILs possess substituents other than a proton (e.g. alkyl group) at the site occupied by the labile proton in analogous PILs.

The existing multiple combinations of cations and anions to make up an IL give them the term of "designer" or "tailor-made" solvents, i.e., an IL can be synthesized for a target application. Some examples of cations and anions that have been used in biomass delignification are given in Figure 1.
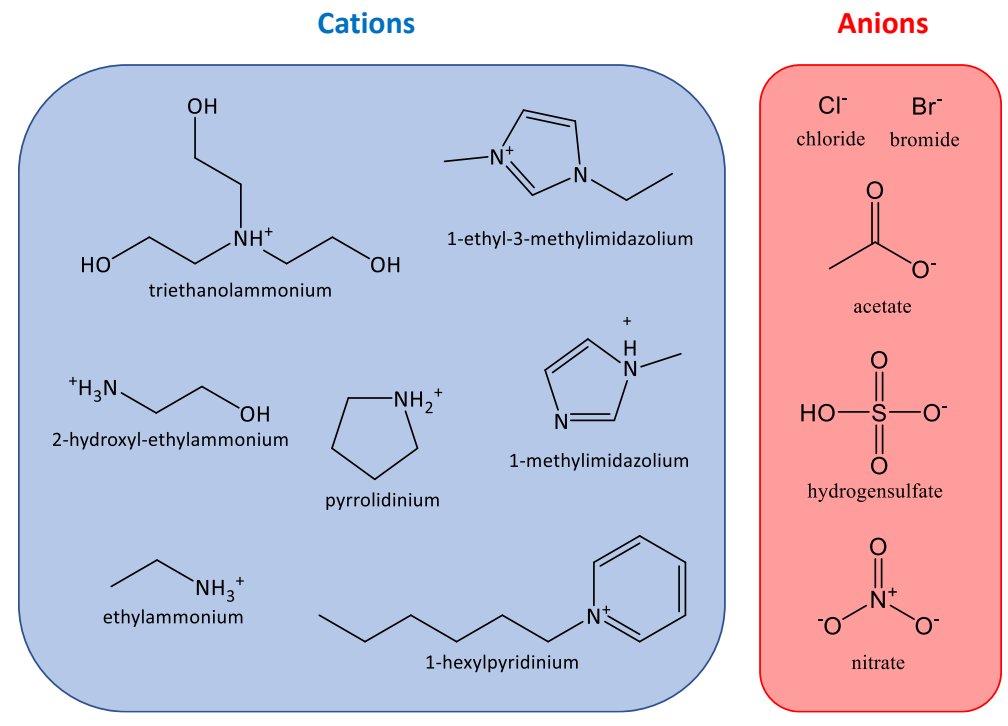

Fig.1. Some examples of cations and anions that make up ionic liquids.

In general, ILs present remarkable features, including negligible vapour pressure, non-flammability, high chemical and thermal stabilities, among others. ILs have been recognized as excellent solvents by taking in account their wide range of polarity, acidity and basicity, which can be tuned by changing the cation or anion in their constitution. Although a definition and general properties can be ascribed to ILs, there are still some exceptions. Some ILs can be volatile, flammable, unstable, and even toxic $[14,15]$. In this regard, a wise choice of IL must be always considered by having in mind the desired application.

In contrast to ILs, DES appears as "juvenile" solvents, since only in the beginning of the present century Abbott et al. have reported them for the first time [16]. Nevertheless, it should be stated that the term eutectic mixture is older. Eutectic mixture refers to the combination of at least two components, generally a hydrogen bond acceptor (HBA) and a hydrogen bond donor (HBD) species, which establish an interaction network (hydrogen bonds and others) capable of decreasing the melting temperature of the mixture (Figure 3 - red line) in comparison with the melting temperature of the individual compounds (Figure 3 - black line as indicative). In other words, a simple mixture of two solids at room temperature may allow the formation of a liquid. An example is given by the solid mixture of urea and a quaternary ammonium salt that turns into a liquid after heating. The addition of the term "deep" to a eutectic mixture relies on a significant deviation of the melting temperature of the mixture (Figure 3 - blue line) when compared to that of an ideal eutectic mixture (Figure 3 red line). In order to identify the eutectic mixture as "deep or not deep" phase diagrams and thermodynamic calculations must be taken in account as reported elsewhere [17]. Although this distinction is important to understand the physicochemical behaviour of the system, most of the times is irrelevant from the application point of view. 


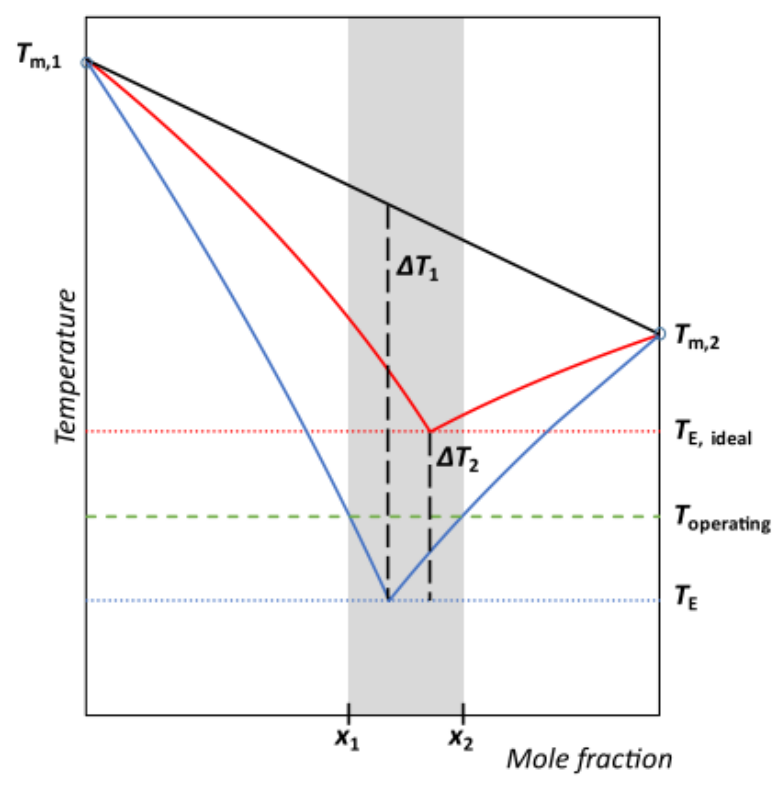

Fig. 2. Schematic representation of solid-liquid equilibria of a simple ideal eutectic mixture (red line) and a deep eutectic mixture (blue line). The black line is just a guide to the eye between melting temperatures of two compounds ( $T_{m, 1}$ and $\left.T_{m, 2}\right) . T_{E}$, ideal = eutectic temperature of an ideal eutectic mixture; $T_{E}=$ eutectic temperature of deep eutectic mixture. Reprinted with permission from [17].

DES (or ES) can be divided into five different categories: Type I and Type II combine a quaternary ammonium salt (usually cholinium chloride $-[\mathrm{Ch}] \mathrm{Cl}$ ) with metal chloride (e.g. ZiCl2) or metal chloride hydrate (e.g. $\mathrm{FeCl} 3 \cdot 6 \mathrm{H}_{2} \mathrm{O}$ ), respectively; Type III relies on the mixture of a quaternary ammonium salt and a HBD (e.g. amides, carboxylic acids, alcohols, among others), which is the most used combination in literature; Type IV is composed of a metal chloride hydrate and HBD; while Type $\mathrm{V}$ is a more recent class and comprises the mixture of non-ionic components, i.e., molecular HBAs and HBDs $[18,19]$. Although hydrogen bonding has been reported as major interaction network existing between DES components (HBA and HBD), and this can be especially true for Type V DES, one should not disregard the ionic bonds in the organic salt(s) composing DES categorized in Types I to IV. There is indeed a distinct chemistry between each DES category and such difference is more relevant when comparing to ILs, which are solely composed of ions (AILs) or mostly composed of ions (PILs). Similar to ILs, the numerous possibilities of combinations to make up DES allow this kind of solvents to be used in a myriad of applications and to be called "designer" or "tailor-made" solvents as well. Some examples of Type III DES components are given in Figure 3. DES share similar features with ILs, including negligible vapour pressure, high chemical and thermal stabilities. Once more, there are exceptions and not all DES exhibit these properties. Moreover, analogous to ILs, a wide range of acidity, basicity, and polarity, among other properties can be tuned by changing DES components and their molar ratios. For this reason, similar applications can be found in literature for both solvents, thus there is quite often the confusion in distinguishing them.

However, there are distinct features that must be highlighted between these two major classes of green solvents. For instance, a comparison of viscosity between two types of most used DES ([Ch]Cl-based) and ILs (imidazoliumbased) was performed elsewhere [20]. DES disclosed a higher viscosity range (0.05 Pa.s up to $2.000 \mathrm{~Pa} \cdot \mathrm{s}$ ) than ILs (0.02 Pa.s up to $7 \mathrm{~Pa} \cdot \mathrm{s})$. The strong hydrogen bonding between [Ch]Cl and its HBD counterpart was mentioned to have higher influence on viscosity than that of cation-anion pair of imidazolium-based ILs. The high viscosity can be an issue associated to DES for industrial applications, particularly those with high number of hydroxyl groups that establish a strong hydrogen bonding network, like sugar-based and polyol-based DES. Although the problem of the high viscosity can be solved quickly by adding water to the system, an excess of water may lead to the disruption of nanostructure lattice of DES. In the application point of view, this fact can be sometimes irrelevant, but a mixture of two components highly diluted in water may not be reasonable. In cases where water plays a crucial role, a simple aqueous solution of the most influent DES component will be more appropriate. Another relevant aspect to distinguish DES and ILs is the preparation of the solvent. DES has been seen as easier to prepare than ILs, which often need complex synthesis, specially AILs as described before. As simple mixture of pure components, DES do not need further purification steps before use. 
Although DES and ILs have been called green solvents mostly because of the negligible vapour pressure, other important environmental factors, such as toxicity and biodegradability, must be addressed as well. Bearing in mind the high number and chemical variability of DES and ILs constituents, it is obvious that these solvents can exhibit high, moderate and low toxicity. Both DES and IL can be toxic depending on the precursor molecules, while sometimes a synergistic effect between components may contribute to higher toxicity than its precursors [21-23] or even lower toxicity [24]. On the other hand, the high stability of these solvents negatively influences its biodegradability. In general, classical imidazolium-based and pyridinium-based ILs are more stable and their biodegradability is difficult. ILs and DES containing natural precursors, like amino acids, sugars, alcohols and organic acids are more biodegradable, thus they could be recommended within an environmental perspective. Furthermore, cholinium-based ILs or [Ch]Cl-based DES are preferred concerning their biodegradability potential. For instance, [Ch]Cl-based DES, such as [Ch]Cl:glycerol (Gly) and [Ch]Cl:oxalic acid (OxA) presented biodegradability yields of $96 \%$ and $68 \%$, respectively [25], while cholinium-based ILs composed of aminoacids (as anion form) showed also good biodegradability levels (62-87 \%) [26]. Nevertheless, in cases where ILs and DES may disclose toxicity and low biodegradability, their full recyclability is of utmost importance, yet a big challenge to surpass. Among several toxicity and biodegradability studies, it is hard to generalize if ILs and DES are non-toxic or biodegradable. There are ILs and DES that present the lowest standards of toxicity and biodegradability, while others exhibit the opposite. Therefore, a case-by-case assessment of the "green" character of these solvents is always required for any chemical process.

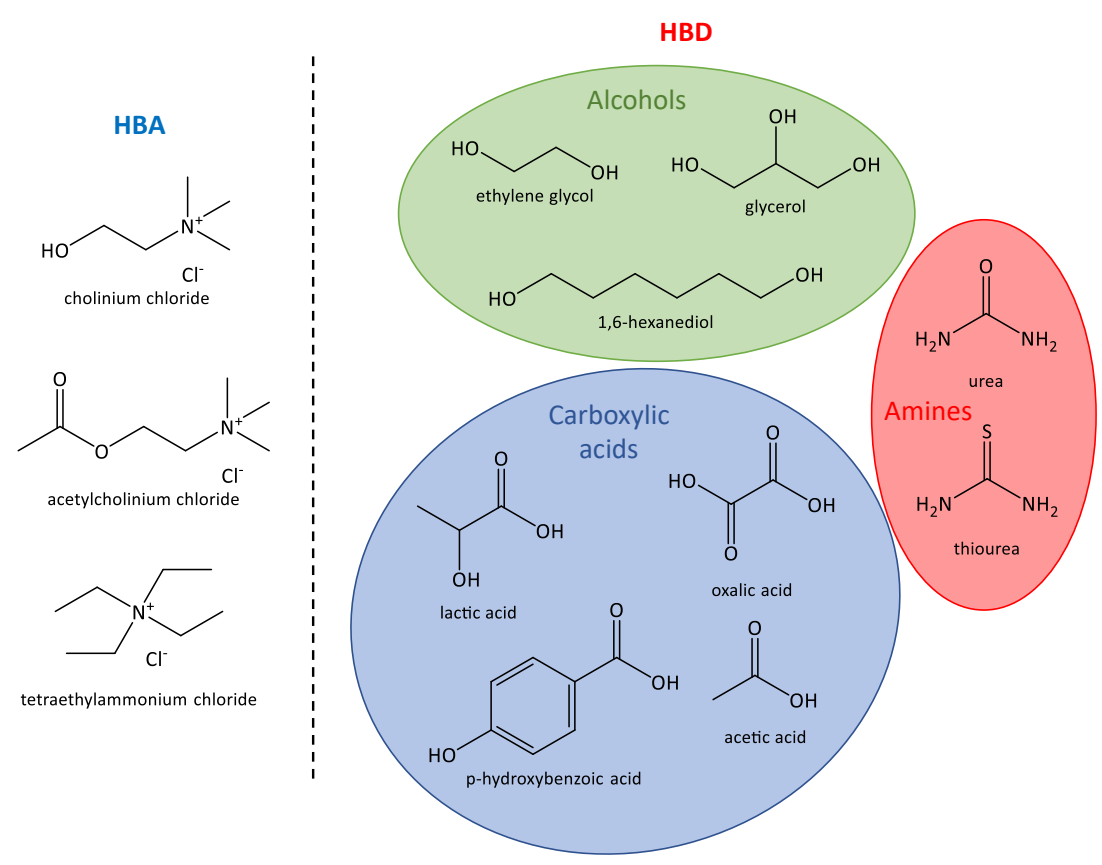

Fig. 3. Some examples of Type III DES possessing a HBA (quaternary ammonium salts) and a HBD (alcohols, carboxylic acids and amines).

Biomass fractionation/delignification with ionic liquids

The application of ILs in lignocellulosic biomass valorisation has slightly more than a decade of research. In 2007, Kilpelainen et al. were the first researchers to demonstrate the ability of ILs, in particular imidazolium-based ILs, to fully dissolve wood at mild conditions $\left(<130^{\circ} \mathrm{C}\right)$ [27]. This work opened the window for a myriad of other studies unveiling the fundamental chemistry behind the IL capacity of interacting with all biomass components and disclosing different strategies for its selective fractionation [28-30]. The mechanisms behind cellulose, hemicellulose and lignin dissolution were mainly ascribed to the ability of ILs to disrupt biomass hydrogen bond matrix and establish new and stronger ones with macromolecular components [31]. In this regard, Remsing et al. reported that IL anion has a more relevant role on the disruption and mediation of hydrogen bonds with biomass polysaccharides than IL cation. In another study, Brandt and co-workers examined the Kamlet-Taft solvatochromic parameters of several ILs and correlated them with the capacity of ILs to dissolve biomass [32]. The researchers found that ILs possessing high basicity anions enables swelling and dissolution of biomass efficiently. Two major examples are the imidazolium-based ILs composed of chloride $\left(\mathrm{Cl}^{-}\right)$and acetate $\left(\mathrm{CH}_{3} \mathrm{COO}\right)$ [33], which among several types of ILs demonstrated the best performance to achieve whole biomass dissolution. 
After biomass dissolution in ILs, the precipitation and regeneration of biomass components can be assisted by the addition of anti-solvents. For instance, cellulose can be regenerated by diluting IL/biomass mixture with water. Basically, water molecules make a hydrodynamic shell around IL ions hindering their interaction with cellulose fibres, which are insoluble in water [33]. This clearly enables the separation of cellulose from other biomass fractions, such as hemicelluloses and lignin. However, the structural characteristics of cellulose suffer a drastic change after regeneration, i.e., the initial semi-crystalline structure shifts to an amorphous-like pulp in the regenerated form. This phenomenon is a technological advantage when targeting the enzymatic digestibility towards glucose by giving more accessible sites to enzymes. However, it is the opposite for paper making, since cellulose loses the desired mechanical properties of their crystalline and well-organized fibres. After cellulose regeneration, hemicelluloses can be precipitated by the addition of ethanol to the resulting liquid medium, while a lignin solid fraction is later obtained by acidification as described elsewhere [30,34]. This selective precipitation and separation of main biomass fractions in a three-step process is one of the advantages of IL technology when benchmarking with conventional fractionation technologies.

As mentioned before, imidazolium-based ILs containing $\mathrm{Cl}^{-}$or $\mathrm{CH}_{3} \mathrm{COO}^{-}$were the most efficient enabling biomass dissolution and further selective precipitation of macromolecular components. However, different works reported that some covalent interactions between imidazolium cation (at C2 position) with cellulose or even degradation into volatile neutral species during the dissolution process may occur leading to IL mass losses in the end of the process $[35,36]$. The fact imidazolium-based ILs are not cheap leads to a negative impact on the total cost of the process. Furthermore, regeneration and reuse of this kind of ILs revealed slight losses of the dissolution performance, caused by biomass components that remain dissolved in the regenerated solvent. Although imidazolium-based ILs and other AlLs capable of dissolving whole biomass have shown efficiency towards biomass fractionation, their high price alongside with mass loss has led to some reluctance among scientific and industry communities to apply them in industrial processes. Furthermore, the use of high amount of water to regenerate and wash cellulose-rich samples can be considered an economic disadvantage.

Therefore, other types of ILs were investigated and those that exhibited more acceptance of application have been PILs, since they are less costly and easier to synthesize than AILs. As mentioned before PILs are synthesised through simple acid-base chemistry between the reaction of a Brønsted acid and a Brønsted base. Acetic, propionic, hexanoic acids are examples of Brønsted acid precursors, while pyridine, pyrrolidine and tertiary amines are examples of Brønsted base precursors used in PIL synthesis. The physicochemical properties of PILs allow different behaviour on the interaction with biomass components in contrast to AlLs. A technical advantage of PILs is their ability towards the selective biomass delignification, or in other words, lignin extraction and dissolution from biomass without dissolving cellulose. This selectivity for lignin has enabled multiple studies with PILs on biomass fractionation as well.

In 2014, Achinivu et al. were the first to demonstrate biomass delignification with PILs, namely pyridinium-based ILs [37]. The researchers obtained approximately $70 \mathrm{wt} \%$ lignin extraction from corn stover after treatment with pyrrolidinium acetate ([Pyrr] $\left.\left[\mathrm{CH}_{3} \mathrm{COO}\right]\right)$ at $90^{\circ} \mathrm{C}$ for $24 \mathrm{~h}$. Among examined cations, pyrrolidinium revealed better performance than pyridinium and imidazolium cations in presence of the counter anion acetate [37]. The use of 2-hydroxyl-ethylammonium (HEA) in combination with acetate also exhibited good performances on lignin extraction after treatment of cashew apple bagasse [38] and sugarcane bagasse [39].These studies revealed that the presence of acetate as the PIL anion showed good performance to enable high biomass delignification yields. Nevertheless, high residence time or high temperature is needed to afford those results.

In a different perspective, Hallett and coworkers demonstrated hydrogen sulfate $\left(\mathrm{HSO}_{4}\right)$-based PILs as both cheap and efficient solvents for biomass delignification. The authors have claimed that mixing sulfuric acid with alkyl amines (e.g. triethylamine or $\mathrm{N}, \mathrm{N}$-dimethylbutylamine) or alkylalcohol amines (e.g. ethanolamine or triethanolamine) allow simple synthesis of PILs reaching a total price around $\$ 1 \cdot \mathrm{Kg}^{-1}$ and reducing the overall costs of the biomass delignification process [40]. These kind of ILs are able to induce biomass delignification (85 $\%)$ at mild conditions $\left(120^{\circ} \mathrm{C}\right)$ [40]. The success of implementing cheap triethylammonium hydrogensulfate ([TEA] $\left[\mathrm{HSO}_{4}\right]$ ) for biomass delignification have also led to the launch of Lixea Ltd. a start-up company that is now running a pilot scale plant to process waste wood, agricultural by-products and sustainably grown biomass with this low cost solvent towards the valorisation of both polysaccharide and lignin fractions into value added compounds [41] The efficiency of $\mathrm{HSO}_{4}$-based ILs on biomass delignification was prior shown by Cox and coauthors, who demonstrated the effective cleavage of $\beta-0-4$ bonds in lignin model compounds by this kind of ILs. 
Similar findings were observed with $\mathrm{Cl}$-based PILs, including 1-methylimidazolium chloride [Hmim][Cl] [42], 1ethylimidazolium chloride [Heim][Cl] [43], 1-hexylpyridinium chloride ([Hpy][Cl]) [44], synthesised through the mixture of hydrochloric acid and corresponding amines.

The fact some PILs can be distilled in the end of the process was pointed out as technical advantage against AILs. The application of heat and vacuum enables the dissociation of the acid-base pair to molecular acid and base precursors, which are then distilled as azeotropes. In the end, the ionicity returns to the regenerated solvent through condensation at low temperature. After biomass delignification, [TEA] [HSO 4 ] can be recovered by distillation up to $99 \%$ [40], while $93 \%$ recovery of [Heim][Cl] was achieved elsewhere [43]. However, this regenerative distillation of PILs cannot be seen as energetically and economically favourable, especially when increasing the scale to biomass tonnes. Indeed, the IL regeneration by precipitation/extraction of dissolved biomass components and further evaporation of anti-solvents, such as water, ethanol and acetone, still continue to be the right decision to do it.

Regarding the quality of isolated lignin after biomass delignification with ILs, the opinions of researchers diverge. In fact, comprehensive data regarding the mechanisms and structural modifications of lignin during biomass dissolution by AILs or biomass delignification with PILs is lacking. Few works however have been unveiling the effects of ILs on lignin structure and overall quality of isolated fraction. Isolated lignin from biomass treatment with 1-ethyl-3-methylimidazolium acetate ([emim] $\left[\mathrm{CH}_{3} \mathrm{COO}\right]$ ) is one of the most studied lignins [45-47]. It is characterised by low chemical and structural modification in contrast to native lignin, although dependent on the severity of biomass treatment. For mild conditions $\left(<120^{\circ} \mathrm{C}\right)$, relatively moderate molecular weight (MW) can be achieved (e.g. $6347 \mathrm{~g} \cdot \mathrm{mol}^{-1}$ ) with narrow polydispersity (PDI <2) [46]. Furthermore, a decrease of the aliphatic $\mathrm{OH}$ content alongside with an increase of phenolic $\mathrm{OH}$ content is generally observed as consequence of dehydration of lignin unit aliphatic chains and $\beta-0-4$ ether bond cleavage, respectively [48]. On the other hand, it seems that $C-C$ bonds, like $\beta-\beta$ and $\beta-5$, are preserved at mild conditions, while at high temperatures (e.g. 160 ${ }^{\circ} \mathrm{C}$ ) this kind of linkages increase due to repolymerization reactions [47]. Other chemical changes in lignin structure, such as demethylation, can be induced by [emim] $\left[\mathrm{CH}_{3} \mathrm{COO}\right.$ ] [48]. All these changes in the isolated lignin seem unanimous in literature when using this IL, yet the mechanisms behind those reactions are still not clear. In addition, it is important to highlight that isolated lignin with [emim] $\left[\mathrm{CH}_{3} \mathrm{COO}\right.$ ] may not be free of xylan impurities as shown in the 2D HSQC spectrum reported elsewhere [47].

On the other hand, the analysis of isolated lignin from biomass delignification with $\mathrm{HSO}_{4}$-based PILs has shown an efficient disruption of hemicellulose-lignin linkages as well as $\beta-0-4$ ether bond cleavage. This is a consequence of the acidic media provided by these ILs [49]. The 2D HSQC spectrum of isolated lignin confirms the absence of $\beta-0-4$ signals as well as xylan and cellulose chemical shifts [50]. Researchers have also found that this type of ILs are able to cleave ether bonds existing in lignin subunits containing $\beta-\beta$ and $\beta-5$ linkages, decreasing their intensity in 2D HSQC spectra [50]. The acidity of $\mathrm{HSO}_{4}$-based PILs also enables the isolation of low $\mathrm{MW}$ lignins when compared [emim] $\left[\mathrm{CH}_{3} \mathrm{COO}\right]$. At long treatments and high temperatures, condensation reactions prevail and substitution reactions on lignin aromatic rings may also occur [50], which must be avoided. However, one important aspect that has not been studied and discussed when using $\mathrm{HSO}_{4}$-based ILs in biomass delignification is the sulfur contamination in isolated lignin fractions. The isolated lignin must be sulfur-free otherwise no technical advantage will be presented against conventional technologies, like Kraft and Sulfite processes.

\section{Biomass delignification with deep eutectic solvents}

The application of DES in biomass fractionation is even more recent. In 2012, the first studies have shown the ability of DES composed of carboxylic acids and [Ch]Cl or aminoacids to dissolve technical lignins [51]. Lignin was dissolved in most of the screened DES, and among them, [Ch]Cl:lactic acid (LA) at molar ratio 1:9 disclosed the highest dissolution performance. In contrast, a high number of DES were examined for cellulose dissolution trials, but the results demonstrated a simple answer: DES are not able to dissolve cellulose [51]. This selectivity for lignin dissolution called the attention of academia and industry, especially the pulp and paper sector, regarding the potential of DES towards selective biomass delignification. In this regard, the Confederation of European Paper Industries (CEPI) chosen deep eutectic solvents (DES) as promising alternative technology to perform a sustainable wood delignification in the future [52]. Since then, several research works dealing with wood and other lignocellulosic biomass delignification processes have been reported [53-57]. 
Although lignin dissolution trials give some perspectives about the required physicochemical properties of DES to perform biomass delignification, their capacity to disrupt the biomass intricate matrix is crucial. For example, the ability of alcohol-based DES, such as [Ch]Cl:glycerol and [Ch]Cl:ethylene glycol, to dissolve high amount of lignin was manifested elsewhere [58]. However, their capacity to extract lignin from biomass is moderate to low. In literature, there are satisfactory biomass delignification yields using these solvents (71-88\%), but this is mostly ascribed to low recalcitrance of studied lignocellulosic materials, such as corncob [54]. For high recalcitrant biomass (e.g. wood) the delignification yields are often lower than $50 \%$. Therefore, these neutral DES disclose low performance to open up the biomass interlinkages and to penetrate and interact efficiently with lignin macromolecules. Similar behaviour is shared by other neutral DES, such as [Ch]Cl:urea (U) [59]. On the other hand, the addition of an acid catalyst (e.g. $0.9 \% \mathrm{H}_{2} \mathrm{SO}_{4}$ ) to alcohol-based DES offers the ability to react with biomass components increasing significantly the delignification yield (up to $80 \%$ after Miscanthus biomass treatment [55]).

In contrast, acid-based DES, including [Ch]Cl:LA or [Ch]Cl:oxalic acid (OxA), not only are effective in dissolving technical lignins but also promote biomass delignification efficiently. The acidity of these solvents is key for the disruption of biomass matrix and cleavage of lignin chemical bonds, particularly $\beta-0-4$ ether bonds, enabling the selective separation of lignin from cellulose fibres. For instance, [Ch]Cl:LA demontrated the highest selectivity for wood delignification by extracting 62 to $84 \%$ of the initial lignin in wood at 130 or $145{ }^{\circ} \mathrm{C}$ for $6 \mathrm{~h}$. AlvarezVasco et al. interrelated the selectivity for delignification mediated by [Ch]Cl:LA with its ability to cleave $\beta-0-4$ ether bonds [56]. Similar to ILs, few studies have addressed the mechanisms behind the interaction and capacity of DES for biomass delignification. Particularly noteworthy, $\mathrm{LA}:[\mathrm{Ch}] \mathrm{Cl}$ revealed a superior delignification than pure lactic acid, suggesting a positive role of $[\mathrm{Ch}] \mathrm{Cl}$ in this process event at low content [60]. It was further demonstrated than in case of acidic [Ch]Cl-based DES the cleavage of $\beta-0-4$ ether bonds is boosted by the presence of the chloride anion [61]. A nucleophile substitution of neighbour hydroxyl groups of $\beta-0-4$ linkages by chloride anion and its subsequent leaving enables a fast formation of enol-ether intermediate that enhances the cleavage [61]. Contrasting to ILs, acid-based DES maintain the integrity of the crystalline structure of cellulose fibres after delignification process, although fibre length is reduced as consequence of acid hydrolysis of cellulose amorphous sections. In this sense, the obtained cellulose fibres reveal lower mechanical performance to produce high quality paper, but it may offer an alternative for other product grades, such as dissolving pulp towards viscose applications.

Although DES have been demonstrated potential for biomass delignification, the viscosity of a large number of DES can be considered as disadvantage as mentioned above. A simple solution comes with the addition of water to reduce the mass transfer limitations. While for some studies the addition of water improved biomass delignification [53], others revealed no change in DES performance (at low water contents) [62]. These differences can be explained by distinct behaviours in the solvation of lignin mediated by DES in presence of water. DES may act as co-solvents, or as hydrotropes enhancing lignin dissolution in aqueous systems significantly $[63,64]$. A good example is [Ch]Cl:1,6-hexanediol that is capable of maintaining maximal Kraft lignin solubility up to $50 \mathrm{wt} \%$ water contents. These results are quite relevant since Kraft lignin solubility in water is negligible. There also cases where the hydrotropic effect exhibited by DES enabled a significant improvement of lignin solubility in aqueous solution when compared to pristine DES [64]. Basically, the presence of hydrophilic water molecules allows stronger interaction between the most hydrophobic regions between lignin and DES [65]. The hydrophobic interactions of both DES and lignin with water are weaker than water-water hydrogen bonds, resulting in strong agglomeration of hydrotropic DES around lignin macromolecules [65]. Recently, the addition of water enhanced the performance of DES in wood delignification, due to the reduction of mass transfer limitations, but also to this hydrotropic mechanism [66].

Another strategy towards biomass delignification is the use of aromatic-based DES. Kim and co-workers demonstrated the ability of DES constituted by lignin derived phenolic compounds, such as catechol, vanillin, $p$ coumaric acid or $p$-hydroxybenzoic acid for biomass delignification [62,67]. One of advantages is the strong $\pi-\pi$ interactions between aromatic rings of solvent and lignin macromolecules favouring lignin extraction from biomass. Up to $70 \%$ lignin removal from woody biomass was achieved with [Ch]Cl: $p$-hydroxybenzoic acid [67]. Although a clear message of sustainability and potential closed-loop biorefinery concept was disclosed by this kind of strategy, the separation of lignin from such solvents is not efficient and leads to significant reduction of DES performance from first use to following recycle runs [67]. 
After biomass delignification, lignin can be easily precipitated from DES liquid stream through the addition of water, which plays as lignin anti-solvent [56]. This precipitation process is more efficient when using acidbased DES. The dilution of this type of DES allows the formation of an aqueous solution with low $\mathrm{pH}$ enhancing the flocculation of previous extracted lignin macromolecules. For neutral DES, acidic water is needed to reach similar precipitation yields. The precipitated lignin has been characterised by different spectroscopic techniques and often highlighted as a high-quality fraction. The selective cleavage of $\beta-0-4$ ether bonds allows an increase of phenolic hydroxyl groups, which means high reactivity properties exhibited by the isolated lignin. Tan et al. studied the variation of phenolic hydroxyl groups of isolated lignins with DES containing different organic acids (e.g. formic, acetic, propionic, lactic, malic, citric, maleic acids, among others) [68]. Among them, [Ch]Cl:LA disclosed the highest yield of phenolic hydroxyl groups in lignin structure and was more efficient than pure lactic acid. Therefore, $[\mathrm{Ch}] \mathrm{Cl}$ seems important not only to speed up the delignification process but also it may offer higher stability to phenolic hydroxyl groups limiting condensation reactions between lignin macromolecules. Moreover, the cleavage of $\beta-0-4$ ether bonds also enables a decrease of the molecular weight in contrast to native lignins. High purity, ranging from $85 \mathrm{wt} . \%$ to $95 \mathrm{wt} . \%$, has been reported for these DES extracted lignins and their use in further applications have been recommended. In addition, these lignins are generally sulphurand carbohydrate-free and show low condensation degree and homogeneous molecular size distribution $[53,56,57]$. These are desired characteristics to enable transformation of lignin in new products and materials. It is unquestionable that the presence of $[\mathrm{Ch}] \mathrm{Cl}$ is significant for delignification efficiency and final characteristics of isolated lignin, thus more in-depth research is needed to unveil the mechanisms behind this delignification and to show the importance of the halide salt/organic acid mixture instead pure or aqueous solution of organic acid (organosolv).

\section{Impact}

The development of novel and innovative processes towards efficient fractionation of lignocellulosic biomass is highly desirable and brings tremendous impact at social, economic and environmental levels. The possibility to produce goods and commodities that we daily know from renewable resources like biomass is expected to progress the sustainability and well fair of world population by disconnecting society from the excessive dependence on pollutant fossil-based resources. On the other hand, the full exploitation of biomass towards energy, fuels, materials and other bio-based products will boost all potentialities of the bioeconomy agenda. This will be achieved when a cascade of fractionation/conversion activities are well designed allowing continuous commercialization of those products. Technical gaps and limitations are still hindering this reality and one of the common drawbacks remains on the disruption of biomass intricate matrix.

The academia and industry have been focused on developing cheap and efficient processes for biomass fractionation, although a duly attention has not been given to selectivity. The selectivity is a crucial aspect that must be optimised to get the full potential of biomass components, namely cellulose, hemicellulose and lignin. A major example lies on biomass delignification step that must address totally or at least a good balance between three important outputs: i) high delignification yield; ii) maintain lignin chemical and structural properties close to native; and iii) avoid degradation of polysaccharides. The existing biomass processing technologies may fail at least one of these outputs. In this sense, the use of tailor-made solvents like ILs and DES with selected physicochemical properties may stand as excellent alternatives. In the last decades of research, they have been showing potential for cheap, effective and sustainable biomass delignification and produce high quality lignin for further conversion and valorisation. Furthermore, not only their performance must be highlighted, but also their eco-friendly benefits. Biomass fractionation and conversion technologies must comprise green chemistry and environmentally friendly practices otherwise no real leverage is gained over conventional petrochemistry activity. In fact, there is a clear advantage in implementing technologies with green solvents to increase process sustainability and to reduce the negative impacts of biomass processing on the environment. ILs and DES have been connoted as such, yet their environmental impact, mostly measured by their toxicity and biodegradability, must be evaluated case-by-case. Moreover, in cases where the technological use of these solvents outperforms any other biomass processing technology, but there will be constraints regarding their toxicity and biodegradability, the full recyclability of the solvent is imperative. There are few studies reporting the recovery of these solvents, although this is a bottleneck that needs much more efforts in research to improve the development and implementation of these technologies in industry.

Therefore, incentives for green chemistry innovations on biomass valorisation must be given, where ILs and DES may play important roles in the future. Among them, ILs are now on the front row to be the next technology for 
biomass fractionation and, as mentioned before, Lixea Ltd. is the first start-up company addressing it. Yet, more incentives are needed to push start-ups and large companies for betting on IL technologies. In case of DES, since limited data is available, more investment in research projects is required to fully understand their potential for biomass fractionation, from fundamentals to application.

\section{Conclusions}

Biomass fractionation should move to another level of development where selectivity must be an important criterion alongside with both performance and ecological efficiencies. In this respect, more selective processes are expected to play an important role to expose cellulose, hemicellulose and lignin fractions separately allowing cascade exploitation of biomass into desired products. This means that the same or higher attention must be given to lignin valorisation as it has been done to polysaccharides, particularly to cellulose. The aromatic character of lignin makes it a potential renewable source of new materials and products, replacing the overexploited aromatic fraction of non-renewable crude oil. In this sense, selective biomass delignification processes are highly required to go beyond the suboptimal use of biomass in conventional processes currently running on bio-based industries, such as pulp and paper industry and $2 \mathrm{G}$ ethanol companies, towards biorefineries.

In this sense, the application of ILs and DES in biomass delignification have been disclosing promising results by enabling higher delignification yields, higher lignin purity and less polysaccharide degradation in comparison to conventional methods. Particularly, AlLs disclose the capacity of dissolving whole biomass to enable further precipitation of major components and subsequent lignin isolation, while both PILs and DES show higher selectivity for lignin extraction and isolation leaving a polysaccharide-rich solid. In general, mild conditions are applied to biomass with these solvents allowing the isolation of lignin fractions with low and narrow molecular weights, low chemical structure modification and free of carbohydrate and sulfur contents. These are relevant physicochemical features that lignin must exhibit for further conversion into chemicals and materials. Yet, more data regarding full chemical and analytical characterisation of isolated lignins, which includes NMR techniques (HSQC, HMBC, ${ }^{13} \mathrm{C}$ NMR and ${ }^{31} \mathrm{P} N M R$ ), GPC, FT-IR, TGA and py-GC/MS analysis, is needed for fair comparison and to address standardization of lignin quality.

Although ILs and DES share similar properties, they also exhibit some differences with significant impact on delignification performance, environmental footprint (toxicity and biodegradability) and solvent recyclability. This makes difficult any kind of generalization to these solvents, thus case-by-case studies must be always assessed. There are still mechanistic insights that need to be unfolded with more in-depth research, while more studies benchmarking biomass delignification using ILs and DES with similar molecular composition are required.

\section{Conflict of interest}

There are no conflicts to declare.

\section{Acknowledgments}

André M. da Costa Lopes thanks his research contract funded by Fundação para a Ciência e Tecnologia (FCT) and project CENTRO-04-3559-FSE-000095 - Centro Portugal Regional Operational Programme (Centro2020), under the PORTUGAL 2020 Partnership Agreement, through the European Regional Development Fund (ERDF). This work was also developed within the scope of the project CICECO-Aveiro Institute of Materials, UIDB/50011/2020 \& UIDP/50011/2020, financed by national funds through the FCT/MCTES.

\section{References}

[1] S.S. Hassan, G.A. Williams, A.K. Jaiswal, Lignocellulosic Biorefineries in Europe: Current State and Prospects, Trends Biotechnol. 37 (2019) 231-234. https://doi.org/10.1016/j.tibtech.2018.07.002.

[2] J.H. Clark, Green biorefinery technologies based on waste biomass, Green Chem. 21 (2019) 1168-1170. https://doi.org/10.1039/c9gc90021g.

[3] P. Gurria, T. Ronzon, S. Tamosiunas, R. Lopez, S. Garcia Condado, J. Guillen, N.E. Cazzaniga, R. Jonsson, M. Banja, G. Fiore, R. M'Barek, Biomass flows in the European Union: The Sankey biomass diagramtowards a cross-set integration of biomass, 2017. https://ec.europa.eu/jrc/en/publication/biomass- 
flows-european-union-sankey-biomass-diagram-towards-cross-set-integration-biomass.

[4] J. Cai, Y. He, X. Yu, S.W. Banks, Y. Yang, X. Zhang, Y. Yu, R. Liu, A. V. Bridgwater, Review of physicochemical properties and analytical characterization of lignocellulosic biomass, Renew. Sustain. Energy Rev. 76 (2017) 309-322. https://doi.org/10.1016/j.rser.2017.03.072.

[5] J.C. Del Río, J. Rencoret, A. Gutiérrez, T. Elder, H. Kim, J. Ralph, Lignin Monomers from beyond the Canonical Monolignol Biosynthetic Pathway: Another Brick in the Wall, ACS Sustain. Chem. Eng. 8 (2020) 4997-5012. https://doi.org/10.1021/acssuschemeng.0c01109.

[6] M. Kuhlberg, T. Särkkä, J. Uusivuori, Technological Transformation in the Global Pulp and Paper Industry: Concluding Remarks, in: Springer, 2018: pp. 279-282. https://doi.org/10.1007/978-3-319-94962-8_13.

[7] J. Wang, L. Wang, D.J. Gardner, S.M. Shaler, Z. Cai, Towards a cellulose-based society: opportunities and challenges, Cellulose. 28 (2021) 4511-4543. https://doi.org/10.1007/s10570-021-03771-4.

[8] P. Bajpai, Pulp and Paper Industry: Energy Conservation, Elsevier B.V., 2016. https://doi.org/10.1016/C2014-0-02105-3.

[9] D. Khatiwada, S. Leduc, S. Silveira, I. McCallum, Optimizing ethanol and bioelectricity production in sugarcane biorefineries in Brazil, Renew. Energy. $85 \quad$ (2016) 371-386. https://doi.org/10.1016/j.renene.2015.06.009.

[10] Z. Sun, B. Fridrich, A. De Santi, S. Elangovan, K. Barta, Bright Side of Lignin Depolymerization: Toward New Platform Chemicals, Chem. Rev. 118 (2018) 614-678. https://doi.org/10.1021/acs.chemrev.7b00588.

[11] B.M. Upton, A.M. Kasko, Strategies for the conversion of lignin to high-value polymeric materials: Review and perspective, Chem. Rev. 116 (2016) 2275-2306. https://doi.org/10.1021/acs.chemrev.5b00345.

[12] M.M. Abu-Omar, K. Barta, G.T. Beckham, J.S. Luterbacher, J. Ralph, R. Rinaldi, Y. Román-Leshkov, J.S.M. Samec, B.F. Sels, F. Wang, Guidelines for performing lignin-first biorefining, Energy Environ. Sci. 14 (2021) 262-292. https://doi.org/10.1039/d0ee02870c.

[13] T. Welton, Ionic liquids: a brief history, Biophys. Rev. $10 \quad$ (2018) 691-706. https://doi.org/10.1007/s12551-018-0419-2.

[14] D. Zhao, Y. Liao, Z.D. Zhang, Toxicity of ionic liquids, Clean - Soil, Air, Water. 35 (2007) 42-48. https://doi.org/10.1002/clen.200600015.

[15] S. Tsuchitani, T. Fukutake, D. Mukai, H. Miki, K. Kikuchi, Unstable Spreading of lonic Liquids on an Aqueous Substrate, Langmuir. 33 (2017) 11040-11046. https://doi.org/10.1021/acs.langmuir.7b01799.

[16] A.P. Abbott, G. Capper, D.L. Davies, R.K. Rasheed, V. Tambyrajah, Novel solvent properties of choline chloride/urea mixtures, Chem. Commun. 1 (2003) 70-71. https://doi.org/10.1039/b210714g.

[17] M.A.R. Martins, S.P. Pinho, J.A.P. Coutinho, Insights into the Nature of Eutectic and Deep Eutectic Mixtures, J. Solution Chem. 48 (2019) 962-982. https://doi.org/10.1007/s10953-018-0793-1.

[18] D.O. Abranches, M.A.R. Martins, L.P. Silva, N. Schaeffer, S.P. Pinho, J.A.P. Coutinho, Phenolic hydrogen bond donors in the formation of non-ionic deep eutectic solvents: The quest for type $v$ des, Chem. Commun. 55 (2019) 10253-10256. https://doi.org/10.1039/c9cc04846d.

[19] E.L. Smith, A.P. Abbott, K.S. Ryder, Deep Eutectic Solvents (DESs) and Their Applications, Chem. Rev. 114 (2014) 11060-11082. https://doi.org/10.1021/cr300162p.

[20] Y.A. Elhamarnah, M. Nasser, H. Qiblawey, A. Benamor, M. Atilhan, S. Aparicio, A comprehensive review on the rheological behavior of imidazolium based ionic liquids and natural deep eutectic solvents, J. Mol. Liq. 277 (2019) 932-958. https://doi.org/10.1016/j.molliq.2019.01.002.

[21] M. Hayyan, M.A. Hashim, A. Hayyan, M.A. Al-Saadi, I.M. AlNashef, M.E.S. Mirghani, O.K. Saheed, Are deep eutectic solvents benign or toxic?, Chemosphere. 90 (2013) 2193-2195. https://doi.org/10.1016/j.chemosphere.2012.11.004.

[22] A. Paiva, R. Craveiro, I. Aroso, M. Martins, R.L. Reis, A.R.C. Duarte, Natural deep eutectic solvents Solvents for the 21st century, ACS Sustain. Chem. Eng. 2 (2014) 1063-1071. https://doi.org/10.1021/sc500096j.

[23] I.P.E. Macário, F. Jesus, J.L. Pereira, S.P.M. Ventura, A.M.M. Gonçalves, J.A.P. Coutinho, F.J.M. Gonçalves, Unraveling the ecotoxicity of deep eutectic solvents using the mixture toxicity theory, Chemosphere. 212 (2018) 890-897. https://doi.org/10.1016/j.chemosphere.2018.08.153.

[24] Z.-L. Huang, B.-P. Wu, Q. Wen, T.-X. Yang, Z. Yang, Deep eutectic solvents can be viable enzyme activators and stabilizers, J. Chem. Technol. Biotechnol. 89 (2014) 1975-1981. https://doi.org/10.1002/jctb.4285.

[25] K. Radošević, M. Cvjetko Bubalo, V. Gaurina Srček, D. Grgas, T. Landeka Dragičević, R.I. Redovniković, Evaluation of toxicity and biodegradability of choline chloride based deep eutectic solvents, Ecotoxicol. Environ. Saf. 112 (2015) 46-53. https://doi.org/10.1016/j.ecoenv.2014.09.034.

[26] X.-D.D. Hou, Q.-P.P. Liu, T.J. Smith, N. Li, M.-H.H. Zong, Evaluation of Toxicity and Biodegradability of 
Cholinium Amino Acids Ionic Liquids, PLoS One. 8 (2013) e59145. https://doi.org/ARTN e59145 DOI 10.1371/journal.pone.0059145.

[27] I.A. Kilpeläinen, H. Xie, A. King, M. Granstrom, S. Heikkinen, D.S. Argyropoulos, Dissolution of wood in ionic liquids, J. Agric. Food Chem. 55 (2007) 9142-9148. https://doi.org/10.1021/jf071692e.

[28] S.P. Magalhães da Silva, A.M. da Costa Lopes, L.B. Roseiro, R. Bogel-Łukasik, Novel pre-treatment and fractionation method for lignocellulosic biomass using ionic liquids, RSC Adv. 3 (2013) 16040-16050. https://doi.org/10.1039/c3ra43091j.

[29] A.M. da Costa Lopes, R.M.G.G. Lins, R.A. Rebelo, R.M. Lukasik, R.M. Łukasik, Biorefinery approach for lignocellulosic biomass valorisation with an acidic ionic liquid, Green Chem. 20 (2018) 4043-4057. https://doi.org/10.1039/c8gc01763h.

[30] A.M. da Costa Lopes, M. Brenner, P. Falé, L.B. Roseiro, R. Bogel-Łukasik, Extraction and Purification of Phenolic Compounds from Lignocellulosic Biomass Assisted by lonic Liquid, Polymeric Resins, and Supercritical CO2, ACS Sustain. Chem. Eng. 4 (2016) 3357-3367. https://doi.org/10.1021/acssuschemeng.6b00429.

[31] A.M. da Costa Lopes, K.G. João, A.R.C. Morais, E. Bogel-Łukasik, R. Bogel-Łukasik, lonic liquids as a tool for lignocellulosic biomass fractionation, Sustain. Chem. Process. 1 (2013) 3. https://doi.org/10.1186/2043-7129-1-3.

[32] A. Brandt, J.P. Hallett, D.J. Leak, R.J. Murphy, T. Welton, The effect of the ionic liquid anion in the pretreatment of pine wood chips, Green Chem. 12 (2010) 672-67. https://doi.org/10.1039/b918787a.

[33] M. Zavrel, D. Bross, M. Funke, J. Büchs, A.C. Spiess, High-throughput screening for ionic liquids dissolving $\begin{array}{lllll}\text { (ligno-)cellulose, } & \text { Bioresour. } & \text { Technol. } & 100 & \text { (2009) }\end{array}$ https://doi.org/10.1016/j.biortech.2008.11.052.

[34] A.M. da Costa Lopes, K.G. João, D.F. Rubik, E. Bogel-Łukasik, L.C. Duarte, J. Andreaus, R. Bogel-Łukasik, Pre-treatment of lignocellulosic biomass using ionic liquids: Wheat straw fractionation, Bioresour. Technol. 142 (2013) 198-208. https://doi.org/10.1016/j.biortech.2013.05.032.

[35] M.T. Clough, K. Geyer, P.A. Hunt, S. Son, U. Vagt, T. Welton, lonic liquids: Not always innocent solvents for cellulose, Green Chem. 17 (2015) 231-243. https://doi.org/10.1039/c4gc01955e.

[36] M.T. Clough, K. Geyer, P.A. Hunt, J. Mertes, T. Welton, Thermal decomposition of carboxylate ionic liquids: Trends and mechanisms, Phys. Chem. Chem. Phys. 15 (2013) 20480-20495. https://doi.org/10.1039/c3cp53648c.

[37] E.C. Achinivu, R.M. Howard, G. Li, H. Gracz, W.A. Henderson, Lignin extraction from biomass with protic ionic liquids, Green Chem. 16 (2014) 1114-1119. https://doi.org/10.1039/c3gc42306a.

[38] C.L.B. Reis, L.M.A. e. Silva, T.H.S. Rodrigues, A.K.N. Félix, R.S. de Santiago-Aguiar, K.M. Canuto, M.V.P. Rocha, Pretreatment of cashew apple bagasse using protic ionic liquids: Enhanced enzymatic hydrolysis, Bioresour. Technol. 224 (2017) 694-701. https://doi.org/10.1016/j.biortech.2016.11.019.

[39] E.G.A. Rocha, T.C. Pin, S.C. Rabelo, A.C. Costa, Evaluation of the use of protic ionic liquids on biomass fractionation, Fuel. 206 (2017) 145-154. https://doi.org/10.1016/j.fuel.2017.06.014.

[40] A. Brandt-Talbot, F.J.V. Gschwend, P.S. Fennell, T.M. Lammens, B. Tan, J. Weale, J.P. Hallett, An economically viable ionic liquid for the fractionation of lignocellulosic biomass, Green Chem. 19 (2017) 3078-3102. https://doi.org/10.1039/c7gc00705a.

[41] F.J.V. Gschwend, L.M. Hennequin, A. Brandt-Talbot, F. Bedoya-Lora, G.H. Kelsall, K. Polizzi, P.S. Fennell, J.P. Hallett, Towards an environmentally and economically sustainable biorefinery: heavy metal contaminated waste wood as a low-cost feedstock in a low-cost ionic liquid process, Green Chem. 22 (2020) 5032-5041. https://doi.org/10.1039/d0gc01241f.

[42] A. Ovejero-Pérez, V. Rigual, J.C. Domínguez, M.V. Alonso, M. Oliet, F. Rodriguez, Acidic depolymerization vs ionic liquid solubilization in lignin extraction from eucalyptus wood using the protic ionic liquid 1methylimidazolium chloride, Int. J. Biol. Macromol. $157 \quad$ (2020) 461-469. https://doi.org/10.1016/j.ijbiomac.2020.04.194.

[43] M.M. Hossain, A. Rawal, L. Aldous, Aprotic vs protic ionic liquids for lignocellulosic biomass pretreatment: Anion effects, enzymatic hydrolysis, solid-state NMR, distillation, and recycle, ACS Sustain. Chem. Eng. 7 (2019) 11928-11936. https://doi.org/10.1021/acssuschemeng.8b05987.

[44] Uju, A. Nakamoto, Y. Shoda, M. Goto, W. Tokuhara, Y. Noritake, S. Katahira, N. Ishida, C. Ogino, N. Kamiya, Low melting point pyridinium ionic liquid pretreatment for enhancing enzymatic saccharification of cellulosic biomass, Bioresour. $\quad$ Technol. $135 \quad$ (2013) $103-108$. https://doi.org/10.1016/j.biortech.2012.06.096.

[45] N. Sathitsuksanoh, K.M. Holtman, D.J. Yelle, T. Morgan, V. Stavila, J. Pelton, H. Blanch, B.A. Simmons, A. 
George, Lignin fate and characterization during ionic liquid biomass pretreatment for renewable chemicals and fuels production, Green Chem. 16 (2014) 1236-1247. https://doi.org/10.1039/c3gc42295j.

[46] J.Y. Kim, E.J. Shin, I.Y. Eom, K. Won, Y.H. Kim, D. Choi, I.G. Choi, J.W. Choi, Structural features of lignin macromolecules extracted with ionic liquid from poplar wood, Bioresour. Technol. 102 (2011) 90209025. https://doi.org/10.1016/j.biortech.2011.07.081.

[47] J.L. Wen, S.L. Sun, B.L. Xue, R.C. Sun, Quantitative structures and thermal properties of birch lignins after ionic liquid pretreatment, J. Agric. Food Chem. 61 (2013) 635-645. https://doi.org/10.1021/jf3051939.

[48] J.L. Wen, T.Q. Yuan, S.L. Sun, F. Xu, R.C. Sun, Understanding the chemical transformations of lignin during ionic liquid pretreatment, Green Chem. 16 (2014) 181-190. https://doi.org/10.1039/c3gc41752b.

[49] A. Brandt, L. Chen, B.E. Van Dongen, T. Welton, J.P. Hallett, Structural changes in lignins isolated using an acidic ionic liquid water mixture, Green Chem. 17 (2015) 5019-5034. https://doi.org/10.1039/c5gc01314c.

[50] F.J.V. Gschwend, C.L. Chambon, M. Biedka, A. Brandt-Talbot, P.S. Fennell, J.P. Hallett, Quantitative glucose release from softwood after pretreatment with low-cost ionic liquids, Green Chem. 21 (2019) 692-703. https://doi.org/10.1039/c8gc02155d.

[51] M. Francisco, A. Van Den Bruinhorst, M.C. Kroon, New natural and renewable low transition temperature mixtures (LTTMs): Screening as solvents for lignocellulosic biomass processing, Green Chem. 14 (2012) 2153-2157. https://doi.org/10.1039/c2gc35660k.

[52] CEPI, Unfold the future. The Two Team Project, 2013.

[53] A.K. Kumar, B.S. Parikh, M. Pravakar, Natural deep eutectic solvent mediated pretreatment of rice straw: bioanalytical characterization of lignin extract and enzymatic hydrolysis of pretreated biomass residue, Environ. Sci. Pollut. Res. 23 (2016) 9265-9275. https://doi.org/10.1007/s11356-015-4780-4.

[54] C.W. Zhang, S.Q. Xia, P.S. Ma, Facile pretreatment of lignocellulosic biomass using deep eutectic solvents, Bioresour. Technol. 219 (2016) 1-5. https://doi.org/10.1016/j.biortech.2016.07.026.

[55] Z. Chen, C. Wan, Ultrafast fractionation of lignocellulosic biomass by microwave-assisted deep eutectic solvent pretreatment, Bioresour. Technol. $250 \quad$ (2018) 532-537. https://doi.org/10.1016/j.biortech.2017.11.066.

[56] C. Alvarez-Vasco, R. Ma, M. Quintero, M. Guo, S. Geleynse, K.K. Ramasamy, M. Wolcott, X. Zhang, Unique low-molecular-weight lignin with high purity extracted from wood by deep eutectic solvents (DES): A source of lignin for valorization, Green Chem. 18 (2016) 5133-5141. https://doi.org/10.1039/c6gc01007e.

[57] X.J. Shen, J.L. Wen, Q.Q. Mei, X. Chen, D. Sun, T.Q. Yuan, R.C. Sun, Facile fractionation of lignocelluloses by biomass-derived deep eutectic solvent (DES) pretreatment for cellulose enzymatic hydrolysis and lignin valorization, Green Chem. 21 (2019) 275-283. https://doi.org/10.1039/c8gc03064b.

[58] F.H.B. Sosa, D.O. Abranches, A.M. Da Costa Lopes, J.A.P. Coutinho, M.C. Da Costa, Kraft Lignin Solubility and Its Chemical Modification in Deep Eutectic Solvents, ACS Sustain. Chem. Eng. 8 (2020) 18577-18589. https://doi.org/10.1021/acssuschemeng.0c06655.

[59] J.L.K. Mamilla, U. Novak, M. Grilc, B. Likozar, Natural deep eutectic solvents (DES) for fractionation of waste lignocellulosic biomass and its cascade conversion to value-added bio-based chemicals, Biomass and Bioenergy. 120 (2019) 417-425. https://doi.org/10.1016/j.biombioe.2018.12.002.

[60] D. Smink, A. Juan, B. Schuur, S.R.A. Kersten, Understanding the Role of Choline Chloride in Deep Eutectic Solvents Used for Biomass Delignification, Ind. Eng. Chem. Res. 58 (2019) 16348-16357. https://doi.org/10.1021/acs.iecr.9b03588.

[61] A.M. Da Costa Lopes, J.R.B. Gomes, J.A.P. Coutinho, A.J.D. Silvestre, Novel insights into biomass delignification with acidic deep eutectic solvents: A mechanistic study of $\beta-0-4$ ether bond cleavage and the role of the halide counterion in the catalytic performance, Green Chem. 22 (2020) 2474-2487. https://doi.org/10.1039/c9gc02569c.

[62] K.H. Kim, T. Dutta, J. Sun, B. Simmons, S. Singh, Biomass pretreatment using deep eutectic solvents from lignin derived phenols, Green Chem. 20 (2018) 809-815. https://doi.org/10.1039/c7gc03029k.

[63] B. Soares, A.J.D. Silvestre, P.C. Rodrigues Pinto, C.S.R. Freire, J.A.P. Coutinho, Hydrotropy and Cosolvency in Lignin Solubilization with Deep Eutectic Solvents, ACS Sustain. Chem. Eng. 7 (2019) 12485-12493. https://doi.org/10.1021/acssuschemeng.9b02109.

[64] B. Soares, D.J.P. Tavares, J.L. Amaral, A.J.D. Silvestre, C.S.R. Freire, J.A.P. Coutinho, Enhanced Solubility of Lignin Monomeric Model Compounds and Technical Lignins in Aqueous Solutions of Deep Eutectic 
Solvents, ACS Sustain. Chem. Eng. $5 \quad$ (2017) 4056-4065. https://doi.org/10.1021/acssuschemeng.7b00053.

[65] D.O. Abranches, J. Benfica, B.P. Soares, A. Leal-Duaso, T.E. Sintra, E. Pires, S.P. Pinho, S. Shimizu, J.A.P. Coutinho, Unveiling the mechanism of hydrotropy: Evidence for water-mediated aggregation of hydrotropes around the solute, Chem. Commun. $56 \quad$ (2020) 7143-7146. https://doi.org/10.1039/d0cc03217d.

[66] B. Soares, A.M. da Costa Lopes, A.J.D. Silvestre, P.C. Rodrigues Pinto, C.S.R. Freire, J.A.P. Coutinho, Wood delignification with aqueous solutions of deep eutectic solvents, Ind. Crops Prod. 160 (2021) 113128. https://doi.org/10.1016/j.indcrop.2020.113128.

[67] Y. Wang, X. Meng, K. Jeong, S. Li, G. Leem, K.H. Kim, Y. Pu, A.J. Ragauskas, C.G. Yoo, Investigation of a lignin-based deep eutectic solvent using $\mathrm{p}$-hydroxybenzoic acid for efficient woody biomass conversion, ACS Sustain. Chem. Eng. 8 (2020) 12542-12553. https://doi.org/10.1021/acssuschemeng.0c03533.

[68] Y.T. Tan, G.C. Ngoh, A.S.M. Chua, Effect of functional groups in acid constituent of deep eutectic solvent for extraction of reactive lignin, Bioresour. Technol. 281 (2019) 359-366. https://doi.org/10.1016/j.biortech.2019.02.010. 